\title{
Designing of CSIW Horn Antenna
}

\author{
Sushma Pandey ${ }^{1}$, Mrs.Rohini A. Deshpande ${ }^{1,}$ Smt. Sulabha Ranade ${ }^{2}$ \\ ${ }^{I}$ (Department of E\&TC, KJ Somaiya College of Engineering, Mumbai University, Mumbai, India) \\ ${ }^{2}$ (SAMEER, IIT Campus, Mumbai, India)
}

\begin{abstract}
In this paper we proposed corrugated SIW horn antenna (CSIW). CSIW technology does not require conducting vias to achieve TE10 type boundary conditions. Here vias are replaced by quarter wavelength microstrip stubs arranged in a corrugated pattern on the edges of the waveguide. We have implemented CSIW technology to design horn antenna, so that CSIW horn antenna will have inherited advantage of conventional horn antenna like low VSWR, High gain, relatively wide bandwidth. For the purpose of simulation HFSS is used. Simulation result for CSIW horn antenna in X band is presented here. The objective of this paper is to provide broad perspective of CSIW horn antenna. CSIW horn antenna provides high gain and directivity with wide bandwidth.
\end{abstract}

Keywords : Substrate Integrated Waveguide (SIW), vias, corrugated Substrate Integrated Waveguide (CSIW), Microstrip stubs.

\section{INTRODUCTION}

The Substrate Integrated Waveguide (SIW) was proposed ten years ago [1]. In its simplest form, this waveguide is composed of two parallel rows of metallic posts inserted in a plated substrate. If the distance between the posts and their diameter are chosen properly, the energy leaking between consecutive posts is negligible, as demonstrated in [2].

System on Substrate is a good example of application of SIW Technology. The planar nature of SIW makes it easy to integrate them with active device [12].

\subsection{Overview of CSIW}

Corrugated SIW is Open-circuit quarter-wave-length microstrip stubs can be used in place of vias to form the sidewalls of the SIW [3].This structure can support TE10 mode and is denoted as corrugated SIW (CSIW) [3]. The CSIW is a periodic structure, and its propagation behavior can be efficiently obtained from the generalized transmission matrix of one period [6].

Whereas $\mathrm{Wu}$ et al. [6] treated the periodic load as zero-dimensional, in this work; the effect of microstrip stub loading is uniformly distributed over the width of the stub in the direction of the waveguide longitudinal axis, thereby acknowledging wave interaction with the stub over its entire width and not just at its midpoint. This is important for closely spaced stubs where the stub width is comparable to the period and has a significant impact on the calculated propagation behavior and reduces the effect of mode-expansion truncation on calculated field plots [12]. The effective width model of Lai et al. [8] was adapted to the CSIW to account for fringing fields at the sides of the host waveguide.

The layout of the CSIW shown in Fig. 1[12] is etched on the top conductor of a substrate of height' h' and relative permittivity of $\varepsilon_{\mathrm{r}}$. The ground plane is on the bottom.

The microstrip open-circuit stubs of length $1_{\text {stubare }}$ quarter wavelength at the band centre frequency, thereby artificially creating electric sidewalls [12].

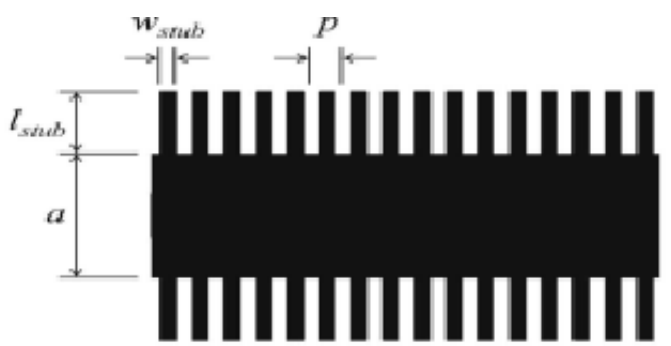

Fig. 1 Corrugated SIW Top conductor layout of the CSIW [12].

The pitch is normally much smaller than the guide wavelength and is similar to vias pitch on SIWs. A stub width $\mathrm{w}_{\text {stub }}$ of was shown to be suitable for an X -band CSIW [3]. 
Corrugated SIW have the two sections a) Waveguide plane which is same as SIW waveguides and b) Corrugations or stub. These stubs are attached to the edge of RSIW upper plane. There is no interconnection between the upper plane and lower plane of RSIW as in vias based RSIW. As per assumption [12] the upper and lower metallic walls of the SIW act as Perfect-Electric-Conductor walls and it has Perfect-Magnetic-Conductor Sidewalls. And the RSIW is considered as dielectric filled waveguide. If the height of the substrate is very less than the wavelength in that dielectric then the waveguide will support TEM and TEm0 modes only to propagate [12].

\subsection{Corrugated SIW horn antenna}

The rectangular waveguide horn is one of the simplest and probably the most widely used microwave antenna. Recently, the substrate-integrated waveguide (SIW) technique has been investigated and developed to construct the planar rectangular waveguide [15]. The application of SIW for the design of an integrated H-plane horn antenna was proposed in [10]. This structure is easy to be integrated with the feed network and is a good candidate to feed the surface-wave antennas or the leaky-wave antennas [11].

Horns provide low VSWR, High gain, relatively wide bandwidth and they are not difficult to fabricate. A rectangular horn is ideally suited for rectangular waveguide feeder. Here, we present a corrugated SIW horn antenna. This antenna is integrated by using a single substrate. It is easy to fabricate and the structure is compact. To eliminate the higher order modes in the waveguide, the thickness of the substrate is restricted. For a horn of maximum gain, the aperture phase distribution along the H-plane is nearly uniform.

HFSS 13 was used to obtain these results. The substrate thickness was $1.6 \mathrm{~mm}$, and its relative permittivity was 3.2 . The CSIW dimensions were $\mathrm{a}=8 \mathrm{~mm}, 1 \mathrm{stub}=4 \mathrm{~mm}$, wstub $=0.5 \mathrm{~mm}$, and $\mathrm{p}=2 \mathrm{~mm}$, and the microstrips taper from 4 to $7 \mathrm{~mm}$ over a length of $8 \mathrm{~mm}$, total length $36 \mathrm{~mm}$.

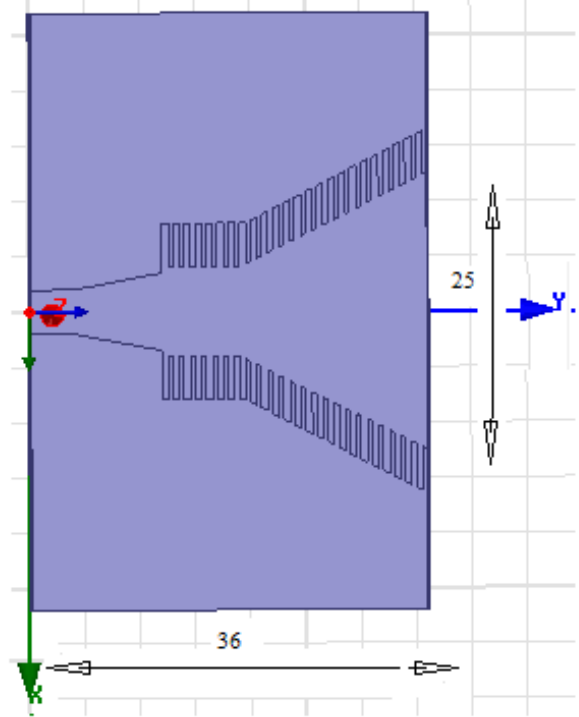

Fig 2 CSIW horn Antenna

\section{THEORETICAL FORMULATION FOR CSIW HORN}

We consider here Rectangular SIW to understand design technique of SIW .Where ' $\mathrm{p}$ ' is the period between the stubs, ' $d$ ' is width of stubs, ' $b$ ' is the height of substrate, 'a' is distance between stubs of both the rows, substrate dielectric constant ' $\varepsilon_{\mathrm{r}}$ ' and ' $\mathrm{a}_{\text {equ }}$ ' is the width of dielectric filled metallic waveguide (equivalent to its vias SIW counterpart).

If ' $\mathrm{a}_{\mathrm{con}}$ ' is the width of conventional wave guide and ' $\varepsilon_{\mathrm{con}}$ ' is 1 (air) than its equivalent dielectric filled waveguide width is formulated as in equation (1):

$$
\mathrm{a}_{\mathrm{equ}}=\mathrm{a}_{\mathrm{con}} / \sqrt{\varepsilon_{\mathrm{r}}}
$$

Depending on ' $\mathrm{a}_{\text {equ }}$ ', where ' $\mathrm{c}$ ' is velocity of light, the cutoff frequency ' $\mathrm{fc}$ ' can be calculated by equation (2):

$$
\mathrm{f}_{\mathrm{c}}=\mathrm{c} /\left(2 . \mathrm{a}_{\text {equ }} \cdot \sqrt{ } \varepsilon_{\mathrm{r}}\right)
$$

The width ' $d$ ' and the periodic distance ' $p$ ' of stubs must be selected such that there are no leakage radiations from them. The periodic length must be greater than the width of the stub so that the structure is physically realizable as in equation (3). 


$$
\mathrm{p}>\mathrm{d}
$$

Since CSIW is a periodic guided wave structure, the electromagnetic band-stop phenomenon will probably appear which should be avoided over the waveguide bandwidth of interest. Therefore the condition shown below (4) should be used to avoid any band-gap effects in the operating bandwidth.

$$
(\mathrm{p} / \lambda \mathrm{g})<0.25
$$

The number of stubs should not exceed 20 per wavelength as in the given condition (5):

$$
(\mathrm{p} / \lambda \mathrm{g})>0.05
$$

The distance between stubs of both the rows ' $a$ ' can be calculated using a simple formula given below (6) and in [14] four other formulas are discussed to determine the value of ' $a$ '.

$$
a=a_{\text {equ }}+\left(d^{2} / 0.95 p\right)
$$

Using the above design techniques or rules we can design a CSIW from its conventional waveguide structure. The stub is the quarter wavelength in length, ' $\mathrm{L}_{\text {stub }}$ '.

$$
\mathrm{L}_{\mathrm{stub}}=\lambda \mathrm{g} / 4
$$

To design the Rectangular CSIW feeder of Horn antenna Design Technique is same as we discussed above. In H-Plane horn antenna the height ' $b$ ' remains same throughout. The Fig 3.2 shows the model structure of Hplane horn antenna.

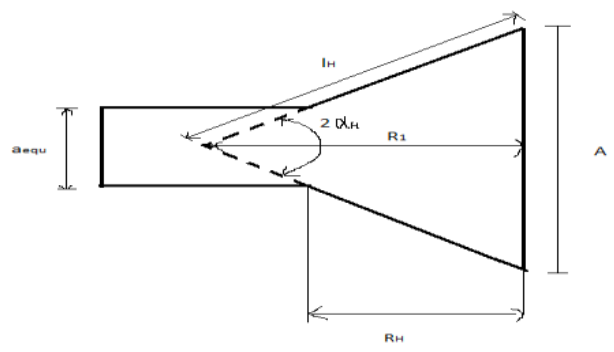

Fig 3 Design of Horn Antenna

The other parameters can be calculated from the relations given below.

$$
\begin{aligned}
& \mathrm{A}=\left(3 \lambda \mathrm{gR}_{1}\right)^{0.5} \\
& \mathrm{l}_{\mathrm{H}}^{2}=\mathrm{R}_{1}^{2}+(\mathrm{A} / 2)^{2} \\
& \alpha_{\mathrm{H}}=\tan ^{-1}\left(\mathrm{~A} / 2 \mathrm{R}_{1}\right) \\
& \mathrm{R}_{\mathrm{H}}=\left(\mathrm{A}-\mathrm{a}_{\text {equ }}\right)\left[\left(\mathrm{l}_{\mathrm{H}} / \mathrm{A}\right)^{2}-0.25\right]^{0.5}
\end{aligned}
$$

The equations (8-11) will help in designing the flare of the horn antenna. Further optimization can be done with respect to the simulation and its result.

\section{SIMULATING RESULT}

Here, we consider a CSIW with a total length of $36 \mathrm{~mm}$, such as no coupling between stubs and uniform current distribution on the stubs, are valid. We are using HFSS-13 as simulator.

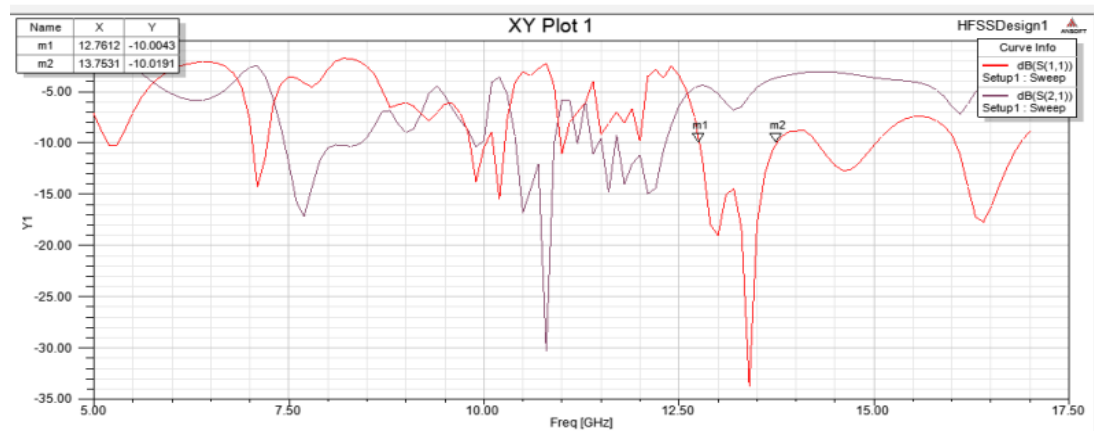

Fig 4 S Parameters of CSIW Horn Antenna at $11 \mathrm{GHz}$ 
First of all we will look at S-Parameters of CSIW Horn Antenna at $11 \mathrm{GHz}$. Here we can see that S-11 is below then $10 \mathrm{~dB}$ between frequency ranges of 12.6 to $13.75 \mathrm{GHz}$. By simulation we are getting wide Band Width of $991.9 \mathrm{KHz}$.

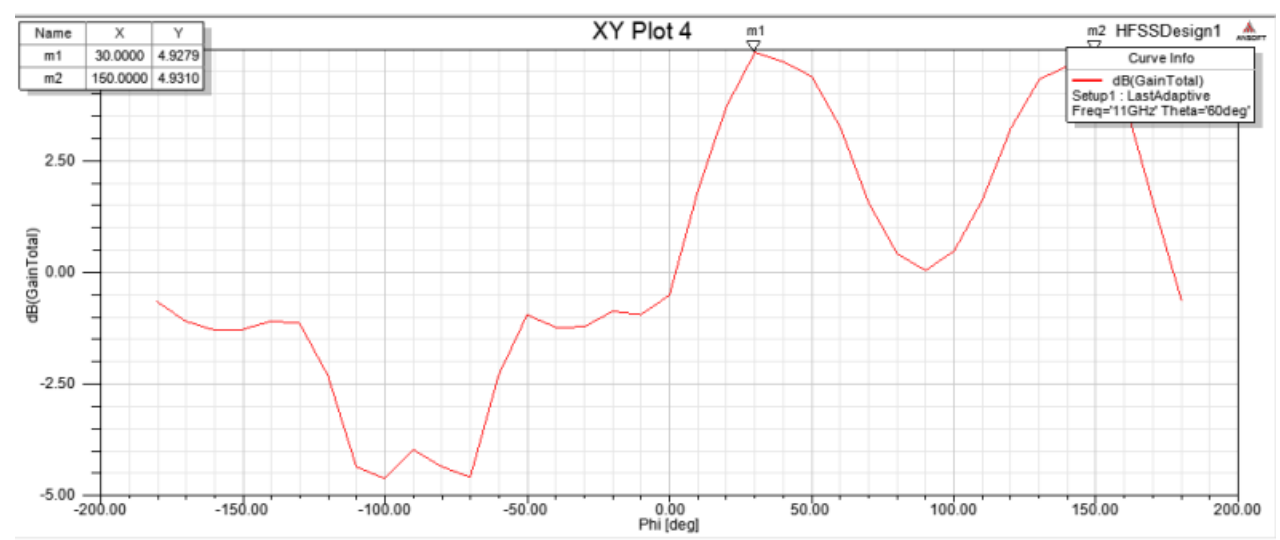

Fig 5 Gain of CSIW Horn Antenna at $11 \mathrm{GHz}$

As we have seen in simulating results of CSIW horn antenna the gain is $5 \mathrm{db}$ at frequency of $11 \mathrm{GHz}$, which is shown in Fig 5.so we are getting high gain along with high bandwidth.



Fig 6 Directivity and beam width of CSIW Horn Antenna at $11 \mathrm{GHz}$

For this antenna the directivity and beam width is as follows fig 6 is showing observation of high directivity but normal beam width of 41.27 .

\section{CONCLUSION}

The CSIW is similar to SIW but uses quarter-wave open-circuit microstrip stubs in place of vias to artificially create electric sidewalls. This structure provides the facility of integration of active devices. In this paper, a CSIW horn antenna has been modeled and using HFSS-13 to get the simulation results. By analysis of results we can say that the advantages of conventional horn antenna like high gain, low beamwidth and low VSWR is continue here with CSIW Horn Antenna. Here we are getting wide bandwidth of $991.9 \mathrm{KHz}$, high gain of $5 \mathrm{~dB}$ and normal beam width of 41.27. This model was validated with simulations of a CSIW designed to operate at the X-band.

\section{ACKNOWLEDGEMENTS}

I would like to thanks the $\mathrm{K} J$ Somaiya College of engineering and SAMEER to providing research opportunity in the field of Antenna.

\section{REFERENCES}

[1] J. Hirokawa and M. Ando, "Single-layer feed waveguide consisting of posts for plane TEM wave excitation in parallel plates," IEEE Trans. Microwave Theory \& Tech., vol. 46, no. 5, pp. 625-630, May 1998.

[2] D. Deslandes and K. Wu, "Accurate modeling, wave mechanism, and design consideration of a substrate integrated waveguide," IEEE Trans. Microwave Theory \& Tech., vol. 54, no. 6,pp. 2516-2526, Jun. 2006.

[3] D. G. Chen and K. W. Eccleston, "Substrate integrated waveguide with corrugated wall," in Proc. Asia-Pacific Microw. Conf., 2008 . 
[4] M. Abdolhamidi, A. Enayati, M. Shahabadi, and R. Faraji-Dana,"Wideband single-layer DC-decoupled substrate integrated waveguide (SIW) - to- microstrip transition using an interdigital configuration," in Proc. Asia-Pacific Microw. Conf., 2007

[5] C. A. Balanis, Antenna Theory: Analysis and Design. New York: Harper and Row, 1982, pp. 579-587.

[6] L.-S. Wu, X.-L.Zhou, W.-Y.Yin, C.-T.Liu, L. Zhou, J.-F.Mao, andH.-L. Peng, "A new type of periodically loaded half-mode substrate integrated waveguide and its applications," IEEE Trans. Microw. Theory Tech., vol. 58, no. 4, pp. 882-893, Apr. 2010.

[7] Q. Lai, C. Fumeaux, W. Hong, and R. Vahldieck, "Characterization of the propagation properties of the half-mode substrate integrated waveguide," IEEE Trans. Microw. Theory Tech., vol. 57, no. 8, pp. 1996-2004, Aug. 2009.

[8] Z. L. Li and K. Wu, "An new approach to integrated horn antenna," inProc. Int. Symp. on Antenna Technology and Applied Electromagnetics, Jul. 2004, pp. 535-538.

[9] Y. Cassivi and K. Wu, "Low Cost Microwave Oscillator Using Substrate Integrated Waveguide Cavity," IEEE Microw. Wireless Compon.Lett., Vol. 13, No. 2, pp. 48-50, Feb. 2003.

[10] M. Abdolhamidi, A. Enayati, M. Shahabadi, and R. Faraji-Dana, "Wideband singlelayer DC-decoupled substrate integrated (SIW) to - microstrip transition using an interdigital configuration," 2007 Asia-Pacific Microwave. Conference, pp. 1599-1602.

[11] C. Zhong, J. Xu, Z. Yu, M. Wang, Y. Zhu, "Half Mode Substrate Integrated Waveguide Gunn Oscillator," Global Symp.onMillimeter Waves Proc. 2008, pp.14-16, 21-24 Apr. 2008.

[12] Kimberley W.Ecclecton, "Mode Analysis of Corrugated Substrate Integrated Waveguide", IEEE Transaction on Microwave Theory and Techniques, Vol 60, No. 10, pp.1-9,New Zealand, October 2012.

[13] FarzanehTaringou and Jens Bornemann, "Return-Loss Investigation of Equivalength width of Substrate Integrted Waveguide Circuits", IEEE MTT-S International Microwave Workshop Series on millimeter Wave Integraton Technologies, pp. 140-143, Canada, September 2011.

[14] Denis G. Chen, and Kimberley W. Eccleston“Substrate Integrated Waveguide with Corrugated Wall', 978-1-4244-26423/08/\$25.00 C2008 IEEE.

[15] Hao Wang, Member, IEEE, Da-Gang Fang, Fellow, IEEE, Bing Zhang, and Wen-QuanChe, Member, IEEE” Dielectric Loaded Substrate Integrated Waveguide(SIW) H-Plane Horn Antennas" IEEE TRANSACTIONS ON ANTENNAS AND PROPAGATION, VOL. 58, NO. 3, MARCH 2010 